\title{
Expression and prognostic value of SFRP1 and $\beta$-catenin in patients with glioblastoma
}

\author{
LIANG CHANG $^{1 *}$, XUHUI LEI $^{2 *}$, YU QIN $^{3}$, GUANGCHUN ZENG $^{3}$, XUEXIN ZHANG $^{1}$,

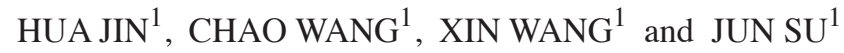 \\ ${ }^{1}$ Department of Neurosurgery, The Tumor Hospital; ${ }^{2}$ Department of Neurosurgery, The Second Affiliated Hospital;
${ }^{3}$ Department of Pathology, The Tumor Hospital, Harbin Medical University, Harbin, Heilongjiang 150086, P.R. China
}

Received October 24, 2014; Accepted September 4, 2015

DOI: $10.3892 / \mathrm{ol} .2015 .3873$

\begin{abstract}
The roles of secreted frizzled-related protein-1 (SFRP1) and $\beta$-catenin in human cancer have been widely studied, and it has recently been demonstrated that these proteins are associated with numerous human carcinomas. However, their clinical significance in glioblastoma multiforme (GBM) has not been examined. The current study aimed to analyze the correlation between the expression of SFRP1 and $\beta$-catenin, and clinicopathological characteristics in GBM patients. The expression of SFRP1 and $\beta$-catenin was assessed by immunohistochemistry in 113 samples of GBM and 40 normal brain tissues. Compared with normal brain tissues, GBM tissues exhibited significantly lower expression of SFRP1, and higher expression of $\beta$-catenin (both $\mathrm{P}<0.05$ ). A Kaplan-Meier analysis revealed that patients with positive SFRP1 expression had a significantly longer overall survival (OS) time relative to those with negative SFRP1 expression $(\mathrm{P}<0.000)$, and that patients with positive $\beta$-catenin expression had a shorter OS time than those with negative $\beta$-catenin expression $(\mathrm{P}<0.000)$. A multivariate $\mathrm{Cox}$ regression analysis indicated that adjuvant treatment, SFRP1 expression and $\beta$-catenin expression were independent prognostic factors for OS $(\mathrm{P}<0.000, \mathrm{P}=0.008$ and $\mathrm{P}=0.001$, respectively) in patients with GBM. The current data suggest that expression of SFRP1 and $\beta$-catenin may be considered significant prognostic indicators for patients with GBM.
\end{abstract}

\section{Introduction}

The most common and devastating of neuroglial tumors is glioblastoma multiforme (GBM), a grade IV astrocytoma (1-3). Despite advanced treatment with surgery, chemotherapy and

Correspondence to: Professor Jun Su, Department of Neurosurgery, The Tumor Hospital, Harbin Medical University, 150 Haping Road, Harbin, Heilongjiang 150086, P.R. China

E-mail: sujun6686@126.com

*Contributed equally

Key words: SFRP1, $\beta$-catenin, glioblastoma, prognosis radiotherapy, the prognosis for patients with GBM remains extremely poor, with a five-year survival rate of $<3 \%(2,3)$. As numerous studies have indicated that complex cellular and molecular events may be involved in oncogenesis and progression of GBM, it is vital to investigate its molecular mechanisms and to identify efficient markers with prognostic and therapeutic significance (1-3).

The Wnt signaling pathway is crucial in the processes of cell proliferation, cell differentiation and embryonic development, and its role in the development of GBM has been previously reported (4). An important downstream component in the canonical Wnt signaling pathway is $\beta$-catenin, which is responsible for the coordination of gene transcription and cell-cell adhesion. The overexpression and mutation of $\beta$-catenin are associated with numerous types of cancer, including endometrial cancer, ovarian cancer and hepatocellular carcinoma (5). When the Wnt protein interacts with Frizzled family receptors to induce the Wnt signaling pathway, $\beta$-catenin accumulates in the cell cytoplasm before translocating into the nucleus to serve as a transcriptional coactivator for transcription factors of the T-cell factor/lymphoid enhancer factor (TCF/LEF) family $(4,6)$. This leads to the activation of downstream target genes, including c-Jun, cyclin D1 and c-Myc (5).

Secreted frizzled-related protein 1 (SFRP1), which maps to chromosome 8p12-p11.1, is the soluble modulator of the Wnt signaling pathway (4-6). SFRP1 interacts with Wnt proteins to prevent them from binding frizzled receptors $(4,6)$. In addition, SFRP1 is able to antagonize Wnt signaling by the formation of an inhibitory complex with frizzled receptors (4). Inactivation of SFRP1 has been reported in various types of malignancy, including gastric cancers, ovarian cancers, prostate cancers, breast cancers, mesothelioma and bladder cancers (6).

However, the significance of SFRP1 and $\beta$-catenin expression in a clinical setting and its prognostic value in GBM remain to be examined. The aim of the current study was to investigate whether SFRP1 was associated with $\beta$-catenin in terms of protein levels, and whether the expression of SFRP1 and $\beta$-catenin had prognostic significance in patients with GBM.

\section{Materials and methods}

Patients and specimens. Approval was obtained from the Ethics Committee of The Tumor Hospital of Harbin Medical 
University (Harbin, China). All patients provided written informed consent. A total of 153 samples were collected from patients of The Tumor Hospital of Harbin Medical University between March 2000 and January 2012, including 113 histologically confirmed GBM samples and 40 normal brain tissue samples. The GBM patients comprised 62 males and 51 females, with a median age of 53.2 years (range, 32-74 years). Patients who had previously received radiotherapy or chemotherapy were excluded. Clinical information for each patient, including gender, age, tumor size, Karnofsky performance status (KPS) (7) score, resection degree and history of adjuvant treatment, was obtained from medical records. All patients completed the follow-up until mortality. During surgical removal of deep benign tumors, normal brain tissue samples were obtained from 24 male and 16 female patients, in the region between the brain surface and the deep brain tumors.

Immunohistochemistry. GBM tissues were fixed in $10 \%$ formalin, embedded in paraffin wax, cut into $4-\mu \mathrm{m}$ sections and mounted on pre-coated slides for use in immunohistochemical analysis. All slides were deparaffinized in xylene (Beyotime Institute of Biotechnology, Haimen, China), rehydrated through a graduated alcohol series and immersed in $3 \% \mathrm{H}_{2} \mathrm{O}_{2}$ (Beyotime Institute of Biotechnology) for $10 \mathrm{~min}$ to inactivate endogenous peroxidases. After washing twice for 5 min with phosphate-buffered saline (PBS; pH 7.2; Beyotime Institute of Biotechnology), the slides were submerged in $0.01 \mathrm{~mol} / \mathrm{l}$ citrate buffer (Beyotime Institute of Biotechnology; $\mathrm{pH}=6.0$ ) for $10 \mathrm{~min}$ and heated at $95^{\circ} \mathrm{C}$ in a microwave oven for antigen retrieval. To reduce non-specific reactivity, $10 \%$ normal goat serum (Bioss, Inc., Beijing, China) was used for $60 \mathrm{~min}$. Subsequently, primary antibodies [polyclonal rabbit anti-human SFRP1 (dilution, 1:100; cat no. AP9037A; Abgent, Inc., San Diego, CA, USA) and monoclonal mouse anti-human $\beta$-catenin (dilution, 1:100; cat no. sc59737; Santa Cruz Biotechnology, Inc., Santa Cruz, CA, USA)] were added separately for overnight incubation at $4^{\circ} \mathrm{C}$. The slides were then incubated with a secondary antibody [biotin-labelled goat anti-mouse (cat no. ZDR-5307) or anti-rabbit antibody (cat no. ZDR-5306); dilution, 1:500; Zhongshan Golden Bridge Biotechnology Co., Ltd., Beijing, China] for $1 \mathrm{~h}$ at room temperature after washing with PBS ( $2 \times 5 \mathrm{~min})$. Thereafter, the slides were stained with 3,3-diaminobenzidine (Zhongshan Golden Bridge Biotechnology Co., Ltd.) for color development. Finally, the slides were counterstained with hematoxylin (Beyotime Institute of Biotechnology), dehydrated and mounted. For the negative control, normal murine IgG (Beyotime Institute of Biotechnology) was used instead of primary antibody.

Evaluation of staining. Immunohistochemically stained slides were viewed separately by two investigators blinded to the clinical information of the patients. Two areas of each slide were randomly selected, from which the number of positively stained cells were counted using a light microscope (DM2700M; Leica Camera AG, Solms, Germany) to determine the percentage of cells expressing each marker. A scoring scale was used to evaluate the percentage of cells stained $(0,0 \% ; 1,1-25 \% ; 2,26-50 \%$; or $3,51-100 \%)$ and
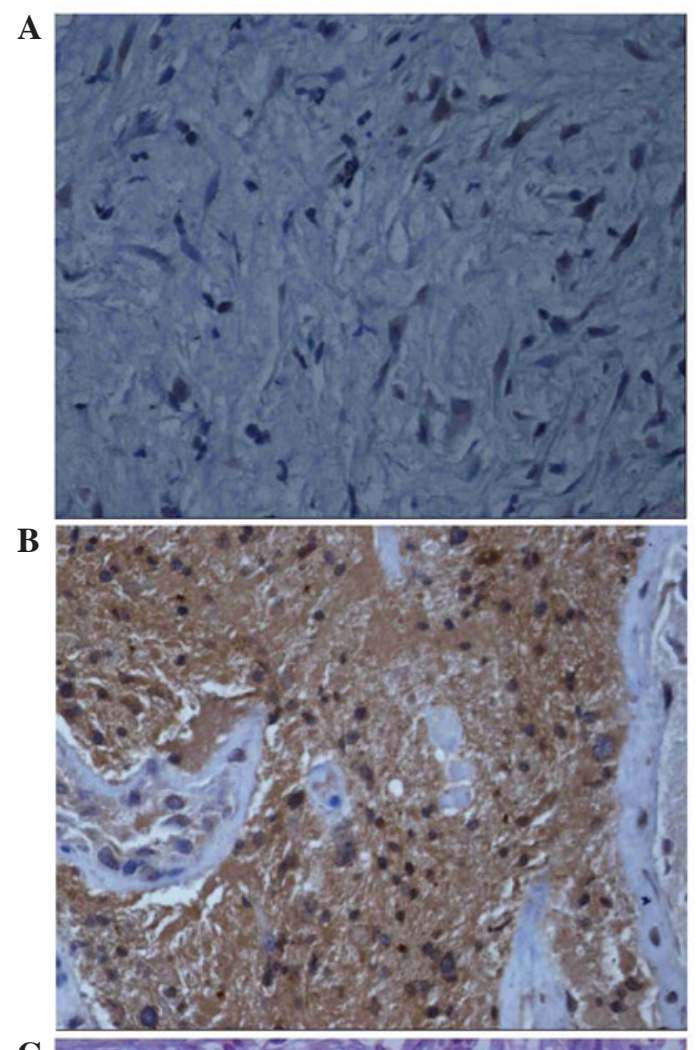

C

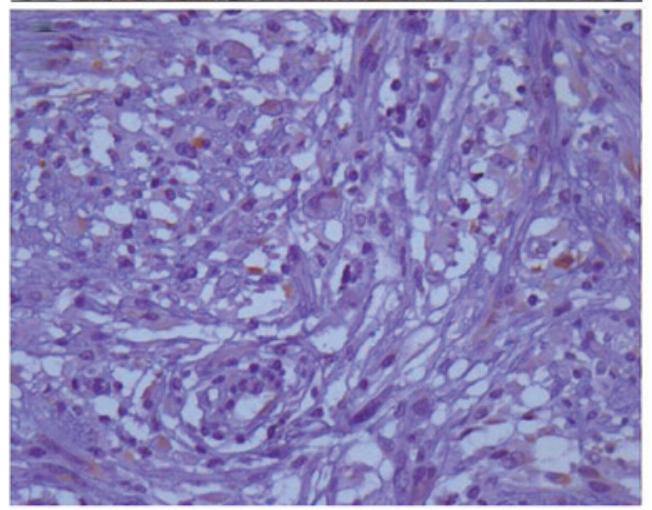

$\mathbf{D}$

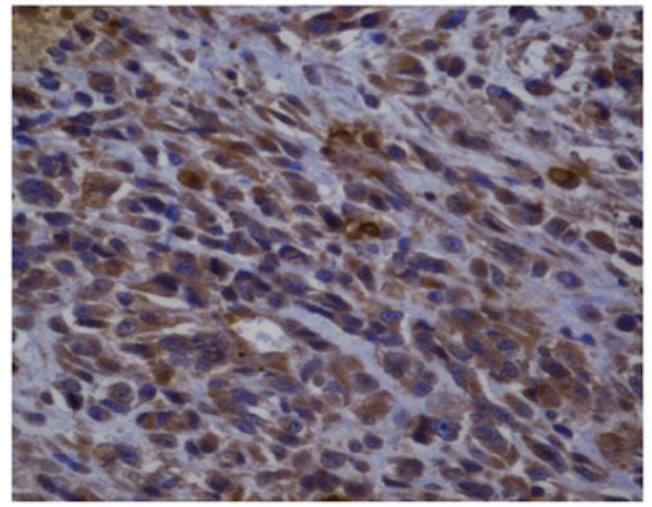

Figure 1. Immunohistochemical scoring pattern of secreted frizzled-related protein-1 (SFRP1) and $\beta$-catenin in glioblastoma. (A) Negative expression of $\beta$-catenin; (B) positive expression of $\beta$-catenin; (C) negative expression of SFRP1; (D) positive expression of SFRP1.

intensity of staining ( 0 , negative; 1 , low; 2 , moderate; 3 , strong). The overall immunohistochemical scores were determined by combining the two staining scores (frequency plus intensity). An immunohistochemical score $\geq 3$ was defined as 
Table I. Associations between the expression of SFRP1, $\beta$-catenin and clinicopathological characteristics in human glioblastoma.

\begin{tabular}{|c|c|c|c|c|c|c|c|}
\hline \multirow[b]{2}{*}{ Variable } & \multirow[b]{2}{*}{$\mathrm{n}$} & \multicolumn{3}{|c|}{ SFRP1 expression, $\mathrm{n}(\%)$} & \multicolumn{3}{|c|}{$\beta$-catenin expression, $\mathrm{n}(\%)$} \\
\hline & & Negative & Positive & P-value & Negative & Positive & P-value \\
\hline Total & 113 & $75(66.37)$ & $38(33.63)$ & & $31(27.43)$ & $82(72.57)$ & \\
\hline Age, years & & & & 0.896 & & & 0.159 \\
\hline$<50$ & 90 & $60(66.67)$ & $30(33.33)$ & & $22(24.44)$ & $68(75.56)$ & \\
\hline$\geq 50$ & 23 & $15(65.22)$ & $8(34.78)$ & & $9(39.13)$ & $14(60.87)$ & \\
\hline Gender & & & & 0.734 & & & 0.011 \\
\hline Female & 51 & $33(64.71)$ & $18(35.29)$ & & $20(39.22)$ & $31(60.78)$ & \\
\hline Male & 62 & $42(67.74)$ & $20(32.26)$ & & $11(17.74)$ & $51(82.26)$ & \\
\hline Tumor size, cm & & & & 0.001 & & & 0.564 \\
\hline$<5$ & 74 & $57(77.03)$ & $17(22.97)$ & & $19(25.68)$ & $55(74.32)$ & \\
\hline$\geq 5$ & 39 & $18(46.15)$ & $21(53.85)$ & & $12(30.77)$ & $27(69.23)$ & \\
\hline KPS score & & & & 0.035 & & & 0.507 \\
\hline$<80$ & 42 & $33(78.57)$ & $9(21.43)$ & & $10(23.81)$ & 32 (76.19) & \\
\hline$\geq 80$ & 71 & $42(59.15)$ & $29(40.85)$ & & $21(29.58)$ & $50(70.42)$ & \\
\hline Resection degree & & & & $<0.001$ & & & $<0.001$ \\
\hline Gross total resection & 64 & $56(87.50)$ & $8(12.50)$ & & $4(6.25)$ & $60(93.75)$ & \\
\hline Partial resection & 34 & $5(14.71)$ & $29(85.29)$ & & $26(76.47)$ & $8(23.53)$ & \\
\hline Biopsy & 15 & $14(93.33)$ & $1(6.67)$ & & $1(6.67)$ & $14(93.33)$ & \\
\hline
\end{tabular}

KPS, Karnofsky performance status; SFRP1, secreted frizzled-related protein-1.

positive expression, whereas a score $\leq 2$ was defined as negative expression.

Statistical analysis. SPSS software version 19.0 (IBM SPSS, Armonk, NY, USA) was used for statistical analysis. The associations between the expression of SFRP1/ $\beta$-catenin and clinicopathological characteristics in GBM patients were assessed using a $\chi^{2}$ test or Fisher's exact probability test. The correlation between SFRP1 and $\beta$-catenin expression was evaluated using Spearman's rank correlation coefficient. The Kaplan-Meier method was used to estimate survival curves and a log-rank test was conducted to evaluate differences in survival distributions. The Cox proportional hazards model was used for the multivariate analyses of prognostic factors. $\mathrm{P}<0.05$ was considered to indicate statistical significance.

\section{Results}

Expression of SFRP1 and $\beta$-catenin in GBM. The levels and patterns of expression of SFRP1 and $\beta$-catenin in GBM tissues and normal brain tissues were examined by immunohistochemical staining. In glioblastoma cells, SFRP1 protein was primarily detected in the cytoplasm, while $\beta$-catenin protein was primarily detected in the cytoplasm and nucleus. Representative images of negative and positive expression of SFRP1 and $\beta$-catenin are shown in Fig. 1 . The rates of positive SFRP1 and $\beta$-catenin expression in GBM were $33.63 \%$ (38/113 samples) and $72.57 \%$ (82/113 samples), respectively. By contrast, normal brain tissue samples exhibited positive expression rates of $77.5 \%$ (31/40 samples) and $17.5 \%$ (7/40 samples) for SFRP1 and $\beta$-catenin, respectively. The
Table II. Association of SFRP1 expression levels and $\beta$-catenin expression status in glioblastoma patients $(n=113)$.

\begin{tabular}{lccccc}
\hline & \multicolumn{4}{c}{$\beta$-catenin expression, $\mathrm{n}$} & \\
\cline { 3 - 4 } $\begin{array}{l}\text { SFRP1 } \\
\text { expression }\end{array}$ & Total & Negative & Positive & P-value & $\mathrm{r}$ \\
\hline Negative & 75 & 14 & 61 & 0.003 & -0.276 \\
Positive & 38 & 17 & 21 & & \\
\hline
\end{tabular}

SFRP1, secreted frizzled-related protein-1.

differences in the rates of expression of the two proteins between GBM samples and normal brain samples were statistically significant $(\mathrm{P}<0.001)$.

Associations between the expression of SFRP1 and $\beta$-catenin and clinicopathological characteristics. As shown in Table I, expression of SFRP1 was significantly associated with tumor size $(\mathrm{P}=0.001)$, KPS score $(\mathrm{P}=0.035)$ and resection degree $(\mathrm{P}<0.000)$. No significant correlation between SFRP1 expression and patient age or gender was found. Regarding $\beta$-catenin, its expression was significantly associated with gender $(\mathrm{P}=0.011)$ and resection degree $(\mathrm{P}<0.000)$ (Table I). However, $\beta$-catenin expression was not significantly associated with age, tumor size or KPS score.

Association of SFRPI expression levels with $\beta$-catenin expression status. Spearman's rank correlation coefficient 
Table III. Multivariate Cox regression analysis of potential prognostic factors for survival of glioblastoma.

\begin{tabular}{|c|c|c|c|c|}
\hline Variable & Comparison & Risk ratio & $95 \% \mathrm{CI}$ & P-value \\
\hline Age, years & $<50$ vs. $\geq 50$ & 0.742 & $0.423-1.303$ & 0.300 \\
\hline Gender & Female vs. male & 1.023 & $0.630-1.659$ & 0.928 \\
\hline Tumor size, mm & $<30$ vs. $\geq 30$ & 1.348 & $0.821-2.213$ & 0.238 \\
\hline KPS score & $<80$ vs. $\geq 80$ & 1.176 & $0.741-1.868$ & 0.491 \\
\hline Resection degree & Gross total resection vs. other & 1.417 & $0.872-2.302$ & 0.159 \\
\hline Adjuvant treatment & Radiotherapy or chemotherapy vs. other & 4.538 & $2.101-9.803$ & $<0.001$ \\
\hline$\beta$-catenin & Negative vs. positive & 2.736 & $1.511-4.952$ & 0.001 \\
\hline SFRP1 & Negative vs. positive & 0.483 & $0.283-0.825$ & 0.008 \\
\hline
\end{tabular}

CI, confidence interval; KPS, karnofsky performance status; SFRP1, secreted frizzled-related protein-1.
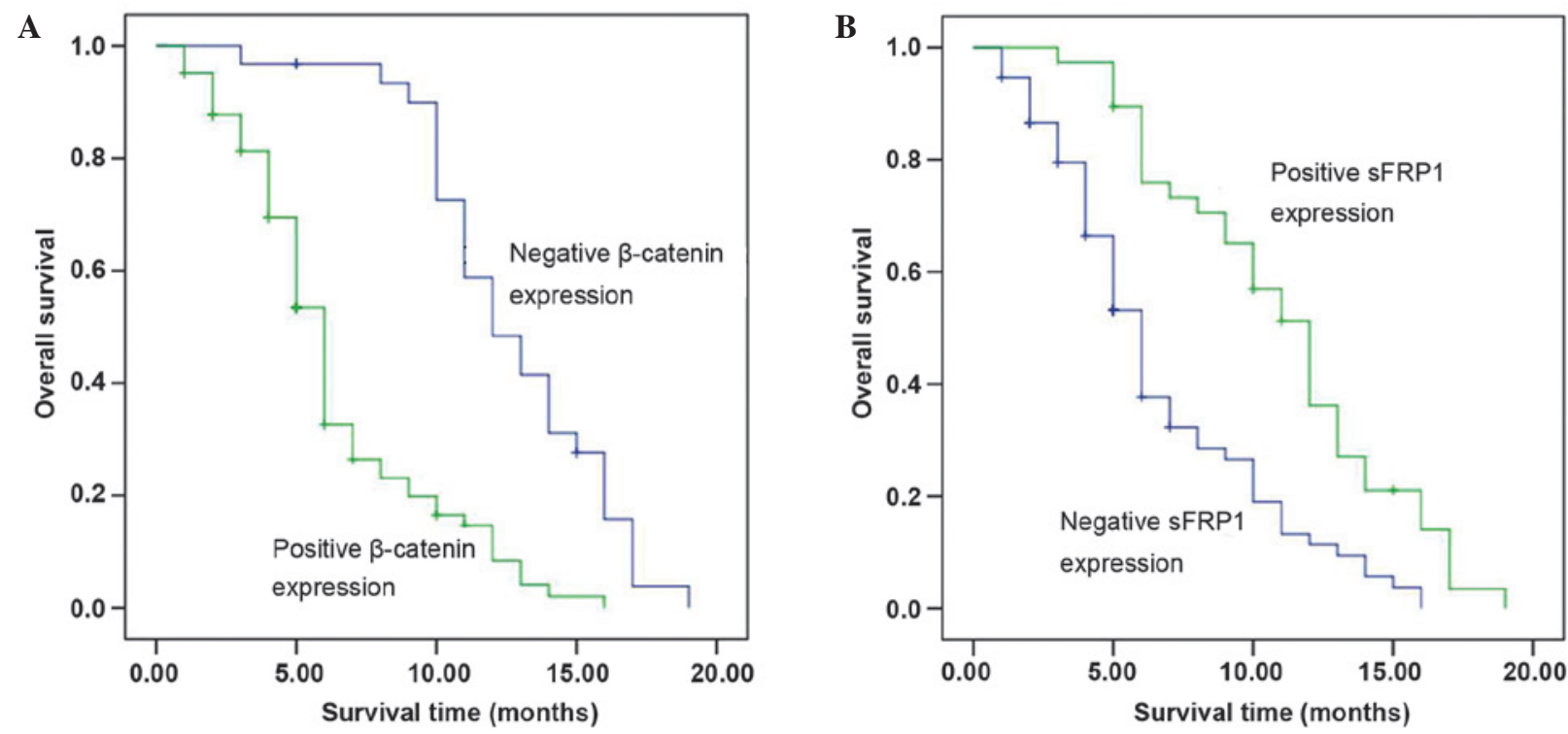

Figure 2. Cumulative survival curves of glioblastoma patients according to expression of (A) $\beta$-catenin protein and (B) secreted frizzled-related protein-1 (SFRP1).

was performed to calculate the association of SFRP1 level with $\beta$-catenin. Of the 75 GBM tissues exhibiting negative expression of SFRP1, 61 tissues exhibited positive expression of $\beta$-catenin. In addition, of the 38 GBM tissues with positive SFRP1 staining, 17 had negative expression of $\beta$-catenin (Table II). Based on Spearman's rank correlation coefficient analysis, there is a close inverse correlation between $\beta$-catenin and SFRP1 expression ( $\mathrm{r}=-0.276, \mathrm{P}=0.003)$.

Prognostic implications of SFRP1 and $\beta$-catenin expression in patients with GBM. The associations of SFRP1 and $\beta$-catenin expression with overall survival (OS) of patients with GBM were analyzed using the Kaplan-Meier method (Fig. 2). The results indicated that patients with positive SFRP1 expression have significantly longer OS times relative to those with negative SFRP1 expression (median, 12 vs. 6 months; $\mathrm{P}<0.000$ ). Accordingly, patients with positive $\beta$-catenin expression have shorter OS time (median, 6 months) relative to those with negative $\beta$-catenin expression (median, 12 months; $\mathrm{P}<0.000$ ).

Furthermore, based on a multivariate Cox regression analysis (which included patient age, gender, tumor size,
KPS score, resection degree, receipt of adjuvant treatment, SFRP1 expression and $\beta$-catenin expression), adjuvant treatment (HR, 5.538; 95\% CI, 2.101-9.803; P<0.000), $\beta$-catenin expression (HR, 2.736; 95\% CI, 1.511-4.952; $\mathrm{P}=0.001)$ and SFRP1 expression (HR, 0.483; 95\% CI, 0.283-0.825; $\mathrm{P}=0.008$ ) were all independent predictors for OS in GBM patients (Table III).

\section{Discussion}

The pathogenesis of GBM is a process of many stages, depending on multiple steps and factors; this includes abnormalities in various cellular adhesive factors, mismatch repair genes, tumor suppressor genes and oncogenes (1-3). SFRP1 and $\beta$-catenin, important members of the Wnt signaling pathway, are involved in numerous cellular processes, including DNA damage response, cell cycle regulation, proliferation, differentiation and apoptosis $(6,8)$. Increasing numbers of studies have provided evidence that SFRP1 and $\beta$-catenin are crucial in the tumorigenesis of a number of malignancies, including non-small-cell lung, hepatocellular, breast and colorectal 
carcinomas (9-12). However, the significance of SFRP1 and $\beta$-catenin expression in the clinical setting and their prognostic value in GBM have not yet been established. Therefore, the current study investigated whether SFRP1 was associated with $\beta$-catenin in terms of protein levels, and whether SFRP1 and $\beta$-catenin levels had prognostic significance in patients with GBM.

$\beta$-catenin maps to chromosome 3p2.1 and was initially identified as an adhesion molecule in 1980 by Walt Bichmeier $(4,6)$. Shortly afterwards, it was found to be a member of the canonical Wnt signaling pathway and serve as a marker of pathway activation (13). The Wnt signaling pathway may be activated through the accumulation and/or altered localization of $\beta$-catenin. Thereafter, $\beta$-catenin forms a complex with the TCF/LEF family of transcription factors, resulting in the activation of growth-controlling genes (14-17). The role of $\beta$-catenin in human carcinomas has been extensively studied (15-17). However, reports regarding the relationship between prognosis and the expression status of $\beta$-catenin in human cancers are limited. Wang et al (16) assessed $\beta$-catenin expression in 60 patients with ovarian serous carcinoma using immunohistochemical staining. This revealed that positive $\beta$-catenin expression was associated with poor prognosis. Similarly, Ji et al (17) assessed $\beta$-catenin mRNA expression levels in 40 esophageal carcinoma patients using reverse transcription-polymerase chain reaction and found that patients with esophageal carcinoma with high $\beta$-catenin expression had a poorer survival rate and a higher rate of lymph node metastasis. In the current study, the positive expression rate of $\beta$-catenin in GBM samples was higher relative to that in normal brain specimens. Furthermore, $\beta$-catenin expression in GBM was also revealed to be associated with patient gender and degree of resection. Based on multivariate analysis, positive $\beta$-catenin expression was a significant independent prognostic factor for reduced OS time in patients with GBM. These results are consistent with those of studies conducted in other cancer types $(16,17)$, suggesting that the Wnt signaling pathway may affect clinical behavior and outcome in patients with GBM.

SFRP1 is a $35 \mathrm{kDa}$ prototypical member of the secreted frizzled-related protein family and maps to chromosome 8p12-p11.1. Silencing of SFRP1 in tumors has been extensively reported, indicating that aberrant inactivation of SFRP1 may be a common mechanism of Wnt signaling activation in solid tumors $(9,11)$. In the present study, the positive rate of SFRP1 expression in glioblastoma samples was lower than that in normal brain specimens, and the protein levels of SFRP1 and $\beta$-catenin were inversely correlated with one another. These findings support the concept of a cancer suppressive role of SFRP1 in human malignancies. Currently, available literature regarding the association between prognosis and the expression status of SFRP1 in human carcinomas is limited. To the best of our knowledge, the present study is the first to investigate this aspect in GBM. The results indicate that SFRP1 expression in GBM is also linked to tumor size, KPS score and degree of resection. According to the multivariate analysis, positive SFRP1 expression was associated with a more favorable prognosis for patients with GBM compared with that of patients with negative SFRP1 expression. These findings indicate that low expression of SFRP1 may promote the progression of GBM. Two possible mechanisms by which low expression of SFRP1 may occur are regulation of microRNA (miR) and methylation of SFRP1 gene promoters. Delic et al (18) demonstrated that SFRP1 was a direct miR-328 target by employing 3' untranslated region luciferase assays as well as miR mimics and inhibitors in glioma cells. Meng et al (19) investigated epigenetic inactivation of the SFRP1 gene in esophageal squamous-cell carcinoma, confirming that complete methylation of the SFRP1 gene promoter was associated with its markedly reduced expression level. Related mechanisms have also been demonstrated in cholangiocarcinoma, lung cancer, prostate cancer, bladder cancer, colon cancer and ovarian cancer (1,20-23). However, future prospective studies are required to further elucidate the efficiency and accuracy of SFRP1 expression in predicting the prognosis of patients with GBM in order to tailor treatment.

In conclusion, the upregulation of $\beta$-catenin protein and downregulation of SFRP1 protein may be important features of GBM. $\beta$-catenin and SFRP1 proteins may be crucial for the progression and prognosis of patients with GBM, indicating that the expression of these proteins may be considered as independent prognostic and early diagnostic markers.

\section{Acknowledgements}

This study was supported by Scientific and Technological Project of Heilongjiang Province of China (GC12C303-1).

\section{References}

1. Yang M, Yuan Y, Zhang H, Yan M, Wang S, Feng F, Ji P, Li Y, Li B, Gao G, et al: Prognostic significance of CD147 in patients with glioblastoma. J Neurooncol 115: 19-26, 2013.

2. Chang KH, Yan MD, Yao CJ, Lin PC and Lai GM: Honokiol-induced apoptosis and autophagy in glioblastoma multiforme cells. Oncol Lett 6: 1435-1438, 2013.

3. Williams M, Tietzel I and Quick QA: 1'-Acetoxychavicol acetate promotes caspase 3 -activated glioblastoma cell death by overcoming enhanced cytokine expression. Oncol Lett 5: 1968-1972, 2013.

4. Wu W, Tian Y, Wan H, Song Y, Li J and Zhang L: The expressions of Wnt/ $\beta$-catenin pathway-related components in brainstem gliomas. Can J Neurol Sci 40: 355-360, 2013.

5. Huo HN, Xie KP, Wang LM, Jiang Y, Zou W and Xie MJ: Relationship between the inhibitory effect of fraxetin on breast cancer and estrogen signaling pathway. Sheng Li Xue Bao 65: 323-328, 2013 (In Chinese)

6. Kang P, Wan M, Huang P, Li C, Wang Z, Zhong X, Hu Z, Tai S and Cui Y: The Wnt antagonist SFRP1 as a favorable prognosticator in human biliary tract carcinoma. PLoS One 9: e90308, 2014.

7. Crooks V, Waller S, Smith T and Hahn TJ: The use of the Karnofsky Performance Scale in determining outcomes and risk in geriatric outpatients. J Gerontol 46: M139-M144, 1991.

8. Lyros O, Rafiee P, Nie L, Medda R, Jovanovic N, Schmidt J, Mackinnon A, Venu N and Shaker R: Dickkopf-1, the Wnt antagonist, is induced by acidic $\mathrm{pH}$ and mediates epithelial cellular senescence in human reflux esophagitis. Am J Physiol Gastrointest Liver Physiol 306: G557-G574, 2014.

9. Stoehr R, Wissmann C, Suzuki H, Knuechel R, Krieg RC, Klopocki E, Dahl E, Wild P, Blaszyk H, Sauter G, et al: Deletions of chromosome 8p and loss of SFRP1 expression are progression markers of papillary bladder cancer. Lab Invest 84 : 465-478, 2004

10. Kocemba KA, Groen RW, van Andel H, Kersten MJ, Mahtouk K, Spaargaren M and Pals ST: Transcriptional silencing of the Wnt-antagonist DKK1 by promoter methylation is associated with enhanced Wnt signaling in advanced multiple myeloma. PLoS One 7: e30359, 2012.

11. Takada T, Yagi Y, Maekita T, Imura M, Nakagawa S, Tsao SW, Miyamoto K, Yoshino O, Yasugi T, Taketani Y and Ushijima T: Methylation-associated silencing of the Wnt antagonist SFRP1 gene in human ovarian cancers. Cancer Sci 95: 741-744, 2004. 
12. Wong CM, Fan ST and Ng IO: beta-Catenin mutation and overexpression in hepatocellular carcinoma: Clinicopathologic and prognostic significance. Cancer 92: 136-145, 2001.

13. Oikonomou E, Barreto DC, Soares B, De Marco L, Buchfelder M and Adams EF: Beta-catenin mutations in craniopharyngiomas and pituitary adenomas. J Neurooncol 73: 205-209, 2005.

14. Salaroli R, Di Tomaso T, Ronchi A, Ceccarelli C, Cammelli S, Cappellini A, Martinelli GN, Barbieri E, Giangaspero F and Cenacchi G: Radiobiologic response of medulloblastoma cell lines: involvement of beta-catenin. J Neurooncol 90 243-251, 2008.

15. Ji H, Wang J, Fang B, Fang X and Lu Z: $\alpha$-Catenin inhibits glioma cell migration, invasion and proliferation by suppression of $\beta$-catenin transactivation. J Neurooncol 103: 445-451, 2011.

16. Wang H, Wang H, Makki MS, Wen J, Dai Y, Shi Q, Liu Q, Zhou $\mathrm{X}$ and Wang J: Overexpression of $\beta$-catenin and cyclinD1 predicts a poor prognosis in ovarian serous carcinomas. Int J Clin Exp Pathol 7: 264-271, 2013.

17. Ji L, Cao XF, Wang HM, Li YS, Zhu B, Xiao J and Wang D: Expression level of beta-catenin is associated with prognosis of esophageal carcinoma. World J Gastroenterol 13: 2622-2625, 2007.

18. Delic S, Lottmann N, Stelzl A, Liesenberg F, Wolter M, Götze S, Zapatka M, Shiio Y, Sabel MC, Felsberg J, et al: MiR-328 promotes glioma cell invasion via SFRP1-dependent Wnt-signaling activation. Neuro Oncol 16: 179-190, 2014.
19. Meng Y, Wang QG, Wang JX, Zhu ST, Jiao Y, Li P and Zhang ST: Epigenetic inactivation of the SFRP1 gene in esophageal squamous cell carcinoma. Dig Dis Sci 56: 3195-3203, 2011.

20. Suzuki H, Gabrielson E, Chen W, Anbazhagan R, van Engeland M, Weijenberg MP, Herman JG and Baylin SB: A genomic screen for genes upregulated by demethylation and histone deacetylase inhibition in human colorectal cancer. Nat Genet 31: 141-149, 2002.

21. Caldwell GM, Jones C, Gensberg K, Jan S, Hardy RG, Byrd P, Chughtai S, Wallis Y, Matthews GM and Morton DG: The Wnt antagonist SFRP1 in colorectal tumorigenesis. Cancer Res 64: 883-888, 2004.

22. Suzuki M, Shigematsu H, Nakajima T, Kubo R, Motohashi S, Sekine Y, Shibuya K, Iizasa T, Hiroshima K and Nakatani Y: Synchronous alterations of Wnt and epidermal growth factor receptor signaling pathways through aberrant methylation and mutation in non small cell lung cancer. Clin Cancer Res 13: 6087-6092, 2007.

23. Sogabe Y, Suzuki H, Toyota M, Ogi K, Imai T, Nojima M, Sasaki Y, Hiratsuka H and Tokino T: Epigenetic inactivation of SFRP genes in oral squamous cell carcinoma. Int J Oncol 32: 1253-1261, 2008. 